\title{
Contingency Ranking With Respect to Overloads in Very Large Power Systems Taking Into Account Uncertainty, Preventive, and Corrective Actions
}

\author{
Stéphane Fliscounakis, Patrick Panciatici, Member, IEEE, Florin Capitanescu, and Louis Wehenkel
}

\begin{abstract}
This paper deals with day-ahead security management with respect to a postulated set of contingencies, while taking into account uncertainties about the next day generation/load scenario. In order to help the system operator in decision making under uncertainty, we aim at ranking these contingencies into four clusters according to the type of control actions needed to cover the worst uncertainty pattern of each contingency with respect to branch overload. To this end we use a fixed point algorithm that loops over two main modules: a discrete bi-level program (BLV) that computes the worst-case scenario, and a special kind of security constrained optimal power flow (SCOPF) which computes optimal preventive/corrective actions to cover the worst-case. We rely on a DC grid model, as the large number of binary variables, the large size of the problem, and the stringent computational requirements preclude the use of existing mixed integer nonlinear programming (MINLP) solvers. Consequently we solve the SCOPF using a mixed integer linear programming (MILP) solver while the BLV is decomposed into a series of MILPs. We provide numerical results with our approach on a very large European system model with 9241 buses and 5126 contingencies.
\end{abstract}

Index Terms-Bi-level programming, mixed integer linear programming, operation under uncertainty, optimal power flow, security-constrained optimal power flow, worst-case analysis.

\section{INTRODUCTION}

\section{A. Motivation}

D AY-AHEAD operational planning, as well as intraday operation of power systems, is affected by an increasing amount of uncertainty due to the coupling of: wind and solar power intermittency, cross-border exchanges, market clearing, load evolution. In this context, a deterministic approach that

Manuscript received July 05, 2012; revised October 22, 2012; accepted November 19, 2012. This paper presents research results of the European FP7 project PEGASE funded by the European Commission. The work of F. Capitanescu was supported by the National Research Fund Luxembourg in the frame of the CORE project "Reliable and efficient distributed electricity generation in smart grids", C11/SR/1278568. The work of L. Wehenkel was supported by the Belgian Network DYSCO (Dynamical Systems, Control, and Optimization), funded by the Interuniversity Attraction Poles Programme, initiated by the Belgian State, Science Policy Office. The scientific responsibility rests with the authors. Paper no. TPWRS-00774-2012.

S. Fliscounakis and P. Panciatici are with the DMA RTE, Versailles, France (e-mail: stephane.fliscounakis@rte-france.com; patrick.panciatici@rte-france. com).

F. Capitanescu is with the Interdisciplinary Centre for Security, Reliability and Trust (SnT), University of Luxembourg, L-1359 Luxembourg (e-mail: florin.capitanescu@uni.lu).

L. Wehenkel are with the Department of Electrical Engineering and Computer Science, University of Liège, B4000 Liège, Belgium (e-mail: 1.wehenkel@ulg.ac.be).

Color versions of one or more of the figures in this paper are available online at http://ieeexplore.ieee.org.

Digital Object Identifier 10.1109/TPWRS.2013.2251015 consists in forecasting a single best-guess of the system injections for the next day or hours, and in ensuring system security along this only trajectory, becomes insufficient.

As the 24-h ahead probabilistic prediction of intermittent renewable sources is not sufficiently accurate, a possible approach to cope with uncertainties consists in checking whether, given some assumptions regarding uncertainties (e.g., defined as active/reactive power injection intervals on buses), the worst case with respect to each contingency is still controllable by appropriate combinations of preventive and corrective actions.

\section{B. Related Work}

To tackle this problem [1] sets up a broader framework as a three-stage decision making process, including slow strategic preventive controls (e.g., starting up a power plant, postponing maintenance works), fast preventive controls (e.g., generation rescheduling) and corrective (or emergency) controls (e.g., generation rescheduling, network switching, phase shifter actions, etc.). The computation of worst-case scenarios is an essential task of this approach.

The worst-case overload condition of a power system under operational uncertainty has been tackled in the literature mostly in the framework of security margins [2], [3]. These approaches tend to compute minimum security margins under operational uncertainty with respect to thermal overloads. These approaches yield min-max optimization problems, since a security margin is, by definition, the maximum value of the loading parameter for a given path of system evolution. However these works do not consider the help of preventive or corrective actions to manage the worst operating states.

Reference [1] formulates the worst case with respect to a contingency as a bi-level (min-max) optimization problem which, assuming a DC load flow approximation and hence focusing on thermal overload only, can be transformed into a mixed integer linear programming (MILP) problem for which suitable solvers are available. [4] proposes an approximate heuristic solution of the bi-level worst-case problem in its nonlinear form (i.e., using the AC network model). Its algorithm relies on the identification in a combinatorial fashion of the worst-case by looking at the sets of constraints violated by worst uncertainty patterns. Furthermore, [8] focuses on the computation of strategic actions in order to cover the worst-cases that cannot be satisfied by best preventive/corrective controls.

\section{Contribution and Organization of the Paper}

In this paper, which builds upon our previous work in [1], we focus on the contingency ranking with respect to overloads in 
very large systems, taking into account uncertainty and preventive/corrective actions. The main contributions of this paper are summarized as follows:

- we implement the iterative algorithm for mixed integer BLV optimization proposed in [6] to compute the worst-case scenario in the presence of corrective actions, whereas in [1] we used an one-step procedure for a continuous BLV;

- we implement a special kind of SCOPF which computes optimal preventive/corrective actions to cover the worst-case, whereas in [1] we computed only the worst-case scenarios;

- we develop a fixed point algorithm [5] to coordinate the convergence of the iterations between these two modules;

- we prove the feasibility of our approach on a very large EHV Pan-European network of 9241 buses and 5126 contingencies, whereas the approach proposed in [1] has been illustrated on a 30-bus system;

- we extend the problem formulation to take into account two types of discrete variables stemming from: control actions with discrete behavior (e.g., fast generators start-up and load shedding) and boolean conditional corrective actions that ensure that a corrective action is triggered only if a certain limit is exceeded.

Note that our problem can be formulated as a very large scale mixed integer nonlinear programming (MINLP). However, nowadays for this class of optimization problem there is no suitable solver - at least given the stringent computational time requirement [7]. For this reason we adopt the DC grid model approximation that allows breaking down significantly the computational time, as our problem is solved by a sequence of very large MILP problems. Besides, this linear approximation has some advantages in terms of desired properties of the optimal solution and accurate network reduction.

We finally notice that other works of the authors [4], [8] utilize the AC nonlinear model as some system operator operational rules (e.g., conditional corrective actions) are relaxed. Consequently, the control variables used were either continuous (e.g., generator active power) or allowed continuous relaxation (e.g., generator start-up as strategic preventive action), enabling thereby the use of a sequence of MILP and NLP solvers. Even under these assumptions, the approaches of [4] and [8] are very computationally intensive on our very large scale model.

The remaining of the paper is organized as follows. Section II provides the main ideas and steps of our approach. Section III formulates the worst-case scenario problem. Section IV presents the two main computational modules of our algorithm: the special kind of SCOPF and the BLV to compute worst-cases. Extensive numerical results with the approach are provided in Section V. Section VI concludes.

\section{RANKING OF CONTINGENCIES INTO FOUR Clusters ACCORding to THEIR SEVERITY}

\section{A. Goals}

In order to check the controllability of postulated contingencies for the worst uncertainty pattern, a very useful result for the system operator ( $\mathrm{SO}$ ) is the ranking of contingencies into four clusters, according to the type of actions needed to cover the worst-case:
TABLE I

FiXed PoInt Algorithm for Covering the Worst-Case of A Single CONTINGEnCY $l$ BY PREVEnTIVE AND CORRECTIVE ACTIONS

do:

1) $\hat{u}_{p}=u_{p}$;

2) $\mathrm{BLV}:$ compute the worst uncertainty pattern $s_{l}\left(\hat{u}_{p}\right)$ after the contingency $l$ in the presence of corrective actions ;

3) SCOPF : compute optimal preventive actions (e.g. minimizing the preventive control change $\left.\left\|u_{p}-\bar{u}_{p}\right\|_{1}\right)$ taking into account corrective actions to cover the worst uncertainty pattern $s_{l}\left(\hat{u}_{p}\right)$ after the contingency $l$.

Let $u_{p}$ be the optimal preventive actions.

while load shedding is not required and $\left\|u_{p}-\bar{u}_{p}\right\|_{2} \geq(1+\epsilon)\left\|\hat{u}_{p}-\bar{u}_{p}\right\|_{2}$

where

- $\bar{u}_{p}$ denotes the given preventive actions ;

- $\epsilon$ is a tolerance for the termination criterion.

1) contingencies which do not require preventive or corrective actions;

2) contingencies which require only corrective actions;

3) contingencies which require corrective and preventive actions;

4) contingencies for which the security of the system cannot be ensured even by the best combination of available preventive and corrective actions.

The SO has to pay attention to contingencies according to the following cluster priority: 4,3 , and 2 .

\section{B. Fixed Point Algorithm for Covering a Single Contingency}

The contingency ranking sought is a by-product of the fixed point algorithm described in Table I, which computes the optimal preventive and corrective actions required to cover the worst-case of a single contingency.

We first solve a bi-level optimization problem in which the preventive controls are frozen in order to assess whether the corrective actions alone are able to ensure the security of the system whatever the uncertainty. Note that, thanks to the definition of the objective function of this bi-level optimization problem, any contingency in clusters 1 and 2 can be detected at the first iteration of the fixed point algorithm and this diagnosis holds whatever the uncertainties.

Then if corrective actions are not sufficient to ensure system security, we solve a special kind of SCOPF problem which computes the optimal combination of preventive and corrective actions to cover the current worst-case scenario. Note that, if best preventive/corrective actions are not sufficient to ensure the system security, case that is revealed by the presence of load shedding, it signifies that the contingency belongs to cluster 4 and we stop. Otherwise, we keep iterating until the convergence of the process, i.e., fixed point on preventive actions, which reveals that the contingency belongs to cluster 3 .

This algorithm involves two major computational steps that we describe mathematically hereafter.

\section{COMPutATion OF THE Worst-CASE Scenario}

\section{A. General Mathematical Formulation of the Problem}

The determination of the worst uncertainty pattern for a contingency requires defining a measure to quantify the worst op- 
erating conditions. A natural choice is to express the worst operating conditions in terms of the maximum overall amount of post-contingency constraint violations (e.g., branch overloads) taking into account corrective actions.

This problem can be formulated as a bi-level mathematical program:

$$
\begin{aligned}
& \delta\left(l, \hat{u}_{p}, S, U_{c}\right)
\end{aligned}
$$

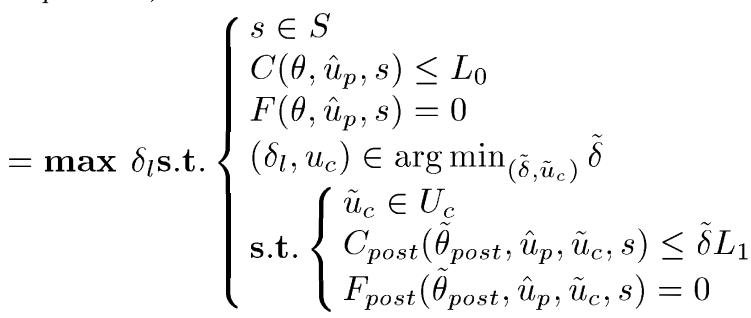

where $s$ is the vector of uncertainty, modeled as bounded active power injections at some buses, $u_{p}$ and $u_{c}$ are vectors of preventive and corrective actions, functions $C$ and $F$ denote the operational constraints and active power flow balance in the base case, functions $C_{\text {post }}$ and $F_{\text {post }}$ denote the operational constraints and active power flow balance after the contingency, $L_{0}$ and $L_{1}>L_{0}$ are base case and post-contingency limits, $\theta$ and $\tilde{\theta}_{\text {post }}$ are voltage angles in the base case and post-contingency, $\delta, \delta, \delta_{l}$ are positive relaxation variables.

Note that in problem (1) the preventive control $\hat{u}_{p} \in U_{p}$ is frozen, and the constraints $C\left(\theta, \hat{u}_{p}, s\right) \leq L_{0}$ and $F\left(\theta, \hat{u}_{p}, s\right)=$ 0 are imposed over the set of possible scenarios, thereby restricting them to those that will lead to realistic and viable precontingency states. As the aim of our approach is to reveal dangerous combinations of uncertainties and contingencies that are difficult or impossible to control with available preventive/corrective actions, we assume that, if needed, preventive controls are set before the application of our approach (e.g., using [4]) so as to avoid overloads in the pre-contingency state $\forall s \in S$.

If the objective of the problem (1) satisfies $\delta\left(l, \hat{u}_{p}, S, U_{c}\right)>$ 1 , it means that for contingency $l$ there are some scenarios for which security cannot be managed only via corrective control. Adjustment of preventive controls are then computed as explained in the algorithm of Table I, based on the most constraining scenario $s_{l}\left(\hat{u}_{p}\right)$ computed by the problem (1).

\section{B. On the Need to Use Discrete Decision Variables}

The adequate modelling of corrective actions, the control rules of some devices, and SO operation rules requires the use of various types of binary variables as follows:

- the start-up of generators to remove post-contingency overloads requires that a generator can be either disconnected from the grid (i.e., $P_{g}=0$ ) or connected to the grid (i.e., $\left.P_{g} \in\left[P_{g}^{\min } ; P_{g}^{\max }\right]\right)$;

- a load can be either connected or fully shed;

- the phase shifter angle change is triggered only if the power flow through the device exceeds its monitored limit.

We emphasize that, unlike the optimization under continuous variables, the presence of these tough discrete variables makes meaningless the information provided by Lagrange multipliers, which justifies our option for the fixed point algorithm.

\section{Some Useful Properties of the DC Approximation}

Thanks to the linear DC approximation the bilevel program (1) has two useful properties:

$$
\begin{aligned}
S^{\prime} \subset S & \Rightarrow \delta\left(l, \hat{u}_{p}, S^{\prime}, U_{c}\right) \leq \delta\left(l, \hat{u}_{p}, S, U_{c}\right) \\
U_{c}^{\prime} \subset U_{c} & \Rightarrow \delta\left(l, \hat{u}_{p}, S, U_{c}^{\prime}\right) \geq \delta\left(l, \hat{u}_{p}, S, U_{c}\right)
\end{aligned}
$$

meaning that shrinking the set of uncertainties (respectively corrective actions) set will lead to smaller (respectively larger) overloads in the worst-case. Note that in the nonlinear case (i.e., AC power system model) these two properties may not hold since a non linear max-min algorithm cannot guarantee the convergence to the global optimum. Moreover, in the DC approximation and in contrast to the $\mathrm{AC}$ model, if an uncertainty vector is feasible for the leader, this uncertainty vector is also feasible for the follower, i.e., after contingency and corrective actions.

\section{Effects of Network Reduction}

We replace the external network by uncertain injections at the boundary nodes, adapting the Ward method [9] to our problem, as detailed hereafter.

From the DC approximation equations $B \theta=P$, the external bus $k$ can be eliminated by using the following formula:

$$
\sum_{j \neq k, i}\left(\frac{B_{i, k} B_{k, j}}{B_{k, k}}-B_{i, j}\right)\left(\theta_{i}-\theta_{j}\right)=P_{i}-\frac{B_{i, k} P_{k}}{B_{k, k}} .
$$

Hence, after elimination of an external zone, the equivalent active powers at boundary buses verify

$$
B^{r} \theta_{i n t}=P_{i n t}+D^{r} P_{e x t}
$$

where superscript $r$ denotes the internal system after reduction, and subscript int (resp. ext) denotes the internal (resp. external) grid. The matrix $D^{r}$ is obtained through an iterative application of formula (4).

Since all entries of matrix $D^{r}$ are non-negative numbers and assuming that all uncertainties in the external network are expressed by nodal injection intervals $\left[P_{e x t}^{\min }, P_{e x t}^{\max }\right]$ one obtains

$$
P_{e x t}^{\min } \leq P_{e x t} \leq P_{e x t}^{\max } \Rightarrow D^{r} P_{e x t}^{\min } \leq D^{r} P_{e x t} \leq D^{r} P_{e x t}^{\max } .
$$

If new uncertainty intervals at boundary nodes are obtained from vectors $D^{r} P_{e x t}^{\min }$ and $D^{r} P_{e x t}^{\max }$, the pessimistic inequality (7) results from property (2):

$$
\delta_{\text {full grid }}\left(l, \hat{u}_{p}, S, U_{c}\right) \leq \delta_{\text {reduced grid }}\left(l, \hat{u}_{p}, S, U_{c}\right) .
$$

So we can reduce parts of the network whose corrective actions $u_{c}$ and flow limits $L_{1}$ have no effect, in such a way as to avoid missing limit violations due to uncertainties. In Appendix A, sufficient conditions will be given to identify these parts.

\section{On the Modeling of SO Operating Rules}

\section{A. SCOPF Modeling SO Operating Rules}

A drawback of the conventional SCOPF [10] is that it does not model the system operator operating rules, which associate 
a pre-defined set of corrective actions with a given post-contingency constraint violation, the corrective actions being activated only if these constraints are violated.

To cover the current worst-case scenario $s_{l}$ of contingency $l$ while taking into account SO operating rules we solve the following SCOPF problem which minimizes the amount of preventive actions:

$$
\begin{aligned}
& \min _{u_{p}, u_{c}, b, d, p, e} w\left\|u_{p}-\bar{u}_{p}\right\|_{1} \\
& \text { s.t. }\left\{\begin{array}{l}
C\left(\theta, u_{p}, s_{l}\right) \leq L_{0} \\
F\left(\theta, u_{p}, s_{l}\right)=0 \\
\left(u_{p}^{\text {min }}-\bar{u}_{p}\right) b \leq u_{p}-\bar{u}_{p} \leq\left(u_{p}^{\text {max }}-\bar{u}_{p}\right) b \\
u_{c}^{\text {min }} e \leq u_{c} \leq u_{c}^{\text {max }} e \\
e \leq R d \quad b, d, p, e \in\{0,1\} \\
-L_{1} \leq M_{l} \theta+M_{c} u_{c} \leq L_{1} \\
-\left(L_{2}+L_{1}\right)(1-p)+L_{1} d \leq M_{l} \theta \leq L_{1}+\left(L_{2}-L_{1}\right) d \\
-\left(L_{2}+L_{1}\right) p+L_{1} d \leq-M_{l} \theta \leq L_{1}+\left(L_{2}-L_{1}\right) d
\end{array}\right.
\end{aligned}
$$

where the preventive actions $u_{p}$ include generation re-dispatch, generator start-up, and load shedding, $u_{p}^{\min }$ and $u_{p}^{\max }$ are bounds on preventive actions, the corrective actions $u_{c}$ include phase shifter angle change and generator start-up, $u_{c}^{\text {min }}$ and $u_{c}^{\max }$ are bounds on corrective actions, $w$ is a vector of weights of preventive control to distinguish between generator re-dispatch or start-up and load shedding in order to ensure that load shedding takes place only if the post-contingency constraints are not met by other types of preventive/corrective actions, binary variables $b$ model the generators start-up and load shedding. Matrix $M_{l}$ links the post-contingency angles to pre-contingency angles $\theta, M_{c}$ is the sensitivity matrix of post-contingency angles to corrective actions, and $L_{2}>L_{1}$ are flow limits that must be satisfied by preventive actions only.

Observe that, thanks to the DC approximation, the constraints after contingency $l$ are expressed implicitly as linear functions of base case angles and corrective actions by means of matrices $M_{l}$ and $M_{c}$.

Boolean matrix $\mathrm{R}$ links post-contingency violated constraints with specific corrective actions, associated to the binary variables $e$, which model the SO operating rules in such a way that $e \leq R d$. Note that the activation of corrective actions according to the SO operating rules, managed by the binary decision variables $d$ and $p$, is described by the set of last five inequalities and operates in the following way. As long as post-contingency flows $M_{l} \theta$ are below their limits $L_{1}$, from the last inequalities we have that $d=0$, which prevent any corrective action $u_{c}=0$. Otherwise, if post-contingency flows $M_{l} \theta$ violate limits $L_{1}$ then $d=1$ and the corresponding corrective actions are activated (i.e., $u_{c}^{\min } \leq u_{c} \leq u_{c}^{\max }$ ) in order to bring flows below limits according to the inequalities $-L_{1} \leq M_{l} \theta+M_{c} u_{c} \leq L_{1}$.

As preventive actions only intervene in the objective function, the optimization naturally uses them only if the full use of corrective actions is insufficient to satisfy post-contingency flow limits.

\section{B. Bilevel Program Modeling Uncertainties and SO Operating Rules}

The mixed-integer bilevel program can be formulated as follows:

$$
\begin{aligned}
& \max _{\theta, s \in S, p \in\{0,1\}, d \in\{0,1\}} \delta_{l} \\
& \left\{\begin{array}{l}
C\left(\theta, \hat{u}_{p}, s\right) \leq L_{0} \\
F\left(\theta, \hat{u}_{p}, s\right)=0
\end{array}\right. \\
& \text { s.t. }\left\{\begin{array}{l}
-\left(L_{2}+L_{1}\right)(1-p)+L_{1} d \leq M_{l} \theta \leq L_{1}+\left(L_{2}-L_{1}\right) d \\
-\left(L_{2}+L_{1}\right) p+L_{1} d \leq-M_{l} \theta \leq L_{1}+\left(L_{2}-L_{1}\right) d \\
\left(\delta_{l}, u_{c}\right) \in \arg \min _{\left(\tilde{\delta}_{l}, \tilde{u}_{c}, \tilde{e}\right)} \tilde{\delta}_{l} \\
\text { s.t. } \\
\left\{\begin{array}{c}
-\tilde{\delta}_{l} L_{1} \leq M_{l} \theta+M_{c} \tilde{u}_{c} \leq \tilde{\delta}_{l} L_{1} \\
u_{c}^{\text {min }} \tilde{e} \leq \tilde{u}_{c} \leq u_{c}^{\max } \tilde{e} \\
\tilde{e} \leq R d \tilde{e} \in\{0,1\}
\end{array}\right.
\end{array}\right.
\end{aligned}
$$

where notations have the same meaning as in the SCOPF problem. The leader (respectively the follower) controls variables are $\theta, s, d, p$ (respectively $\tilde{\delta}_{l}, \tilde{u}_{c}, \tilde{e}$ ).

Note that assuming that all discrete variables $d, \tilde{e}$ are fixed enables us to compute bounds for the variables in the dual problem of the follower. We provide a proof in the Appendix B that these bounds depend only on $L_{1}, L_{2}, u_{c}^{\min }, u_{c}^{\max }$, property which we call hereafter "dual property". As a consequence, we show in the Appendix that the resolution of the corresponding continuous linear bilevel program is equivalent to a classical MILP problem. To solve in the general case the problem (9), we use the method proposed in [6], whose the main steps are presented in the next section, and that can be summarized as a successively tighter lower bounding procedure. To implement the method, the "dual property" is again useful since it enables the use of KKT necessary conditions through big-M formulation for the lower-level problem where discrete variables are fixed.

\section{Discrete Bilevel Programming Algorithm}

We compute the worst uncertainty pattern for a given contingency of the formulation (1) using an iterative algorithm for discrete bi-level programming proposed in [6] which we describe hereafter.

For the sake of clarity and formulation compactness we adopt the following generic notations and make the link with the optimization variables of the previous formulations:

$X^{u} \quad$ set of upper continuous variables $\left.\left(=\left\{\theta, \hat{u}_{p}, s\right\}\right)\right)$;

$Y^{u} \quad$ set of upper discrete variables $(=\{p, d\})$;

$X^{l} \quad$ set of lower continuous variables $\left(=\left\{\tilde{\delta}_{l}, \tilde{u}_{c}\right\}\right)$;

$Y^{l} \quad$ set of lower discrete variables $(=\{\tilde{e}\})$;

$g^{u} \quad$ continuous functions on $X^{u} \times Y^{u}$;

$g^{l, 1} \quad$ continuous functions on $X^{u} \times Y^{u} \times X^{l} \times Y^{l}$;

$g^{l, 2} \quad$ continuous functions on $X^{l} \times Y^{l}$.

The continuity on $g^{u}, g^{l, 1}$ and $g^{l, 2}$ is only required when discrete variables are fixed. 


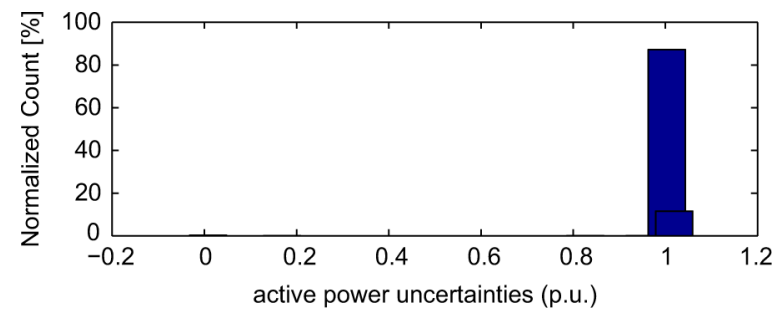

Fig. 1. Algorithm of [6].

A bilevel program with both discrete and continuous variables can be formulated in a generic and compact way as follows:

$\min f^{u}\left(x^{u}, y^{u}, x^{l}, y^{l}\right)$

$$
\text { s.t. }\left\{\begin{array}{l}
g^{u}\left(x^{u}, y^{u}, x^{l}, y^{l}\right) \leq 0 \\
x^{u} \in X^{u}, y^{u} \in Y^{u} \\
\left(x^{l}, y^{l}\right) \in \arg \min _{\left(x^{m}, y^{m}\right)} f^{l}\left(x^{u}, y^{u}, x^{m}, y^{m}\right) \\
\text { s.t. }\left\{\begin{array}{l}
g^{l, 1}\left(x^{u}, y^{u}, x^{m}, y^{m}\right) \leq 0 \\
g^{l, 2}\left(x^{m}, y^{m}\right) \leq 0 \\
x^{m} \in X^{l}, y^{m} \in Y^{l}
\end{array}\right.
\end{array} .\right.
$$

The algorithm in [6] solves a sequence of single-level MILP optimization problems that compute upper bounds (UBD) and lower bounds (LBD) for the original BLV until the difference between these bounds falls below a tolerance $\bar{\epsilon}_{f}^{u}$. This tolerance $\bar{\epsilon}_{f}^{u}$ must verify: $\epsilon_{\text {solver }}<\bar{\epsilon}_{f}^{u}$ where $\epsilon_{\text {solver }}$ is the precision on the objective values associated to the global solutions provided by the classical mixed-integer linear solver chosen to solve the sub-problems described below. Fig. 1 shows the flowchart of the algorithm.

The main challenge of this algorithm is the influence of upper-level variables on the generation of parametric upper bounds for the lower-level program.

The main computational steps of this algorithm are described hereafter. Given the high complexity of the method we refer the reader to [6] for further details and proofs about the method.

1) Parametric Upper Bounds: These bounds are based on pairs comprising a subset of the upper-level host set $X^{u, k} \times Y^{u, k}$ and a lower-level point $\left(x^{l, k}, y^{l, k}\right)$ such that $g^{l, 2}\left(x^{l, k}, y^{l, k}\right) \leq 0$, that remains feasible in the lower-level program for all $\left(x^{u}, y^{u}\right) \in X^{u, k} \times Y^{u, k}$

$$
g^{l, 1}\left(x^{u}, y^{u}, x^{l, k}, y^{l, k}\right) \leq 0 .
$$

2) Lower Bounding: Lower bounds are computed from the following problem:

$$
\begin{aligned}
L B D= & \min f^{u}\left(x^{u}, y^{u}, x^{l}, y^{l}\right) \\
\text { s.t. } & \left\{\begin{array}{l}
g^{u}\left(x^{u}, y^{u}, x^{l}, y^{l}\right) \leq 0 \\
g^{l, 1}\left(x^{u}, y^{u}, x^{l}, y^{l}\right) \leq 0 \\
g^{l, 2}\left(x^{l}, y^{l}\right) \leq 0 \\
x^{u} \in X^{u}, y^{u} \in Y^{u}, x^{l} \in X^{l}, y^{l} \in Y^{l} \\
\forall k \in K, \quad\left(x^{u}, y^{u}\right) \in X^{u, k} \times Y^{u, k} \\
\Rightarrow f^{l}\left(x^{u}, y^{u}, x^{l}, y^{l}\right) \leq f^{l}\left(x^{u}, y^{u}, x^{l, k}, y^{l, k}\right) .
\end{array}\right.
\end{aligned}
$$

TABLE II

PEGASE PAN EUROPEAN EHV NETWORK

\begin{tabular}{ccc}
\hline voltage level (kV) & number of buses number of lines \\
\hline 750 & 3 & 2 \\
400 & 126 & 217 \\
380 & 1814 & 4034 \\
330 & 2 & 1 \\
220 & 3185 & 5638 \\
154 & 728 & 1268 \\
150 & 1530 & 2033 \\
120 & 10 & 13 \\
110 & 1843 & 2764 \\
\hline
\end{tabular}

Using the solution $\left(\bar{x}^{u}, \bar{y}^{u}\right)$ of the lower bounding problem (12), an approximation of the optimal lower objective function is obtained by

$$
\begin{array}{r}
f^{l, *}=\min f^{u}\left(\bar{x}^{u}, \bar{y}^{u}, x^{l}, y^{l}\right) \\
\text { s.t. }\left\{\begin{array}{l}
g^{l, 1}\left(\bar{x}^{u}, \bar{y}^{u}, x^{m}, y^{m}\right) \leq 0 \\
g^{l, 2}\left(x^{m}, y^{m}\right) \leq 0 \\
x^{m} \in X^{l}, y^{m} \in Y^{l}
\end{array}\right.
\end{array}
$$

3) Upper Bounding: Upper bounds are computed from the following problem:

$$
\begin{array}{r}
U B D=\min f^{u}\left(\bar{x}^{u}, \bar{y}^{u}, x^{l}, y^{l}\right) \\
\text { s.t. }\left\{\begin{array}{l}
g^{u}\left(\bar{x}^{u}, \bar{y}^{u}, x^{l}, y^{l}\right) \leq 0 \\
g^{l, 1}\left(\bar{x}^{u}, \bar{y}^{u}, x^{l}, y^{l}\right) \leq 0 \\
g^{l, 2}\left(x^{l}, y^{l}\right) \leq 0 \\
f^{l}\left(\bar{x}^{u}, \bar{y}^{u}, x^{l}, y^{l}\right) \leq \bar{f}^{l, *}+\epsilon_{f}^{l} \\
L B D \leq \bar{f}^{u}\left(\bar{x}^{u}, \bar{y}^{u}, x^{l}, y^{l}\right) \\
x^{l} \in X^{l}, y^{l} \in Y^{l}
\end{array}\right.
\end{array}
$$

\section{Numerical Results}

\section{A. Description of the PEGASE Pan European EHV Network}

We use a very large scale modified model of the interconnected EHV European power system which spans from Portugal and Spain to Ukraine, Russia, Greece and Turkey. Notice that in this model the real parameters of the individual power systems components (e.g., lines, transformers, etc.), the network topology, as well as the limits on: generators active/reactive powers, transformers ratio and angle, and branch currents have been biased. Nevertheless, this model is representative of the European system in terms of size and complexity. Furthermore, we have chosen very tight operational limits and physical bounds of controls in order to assess the robustness of our tools. For instance the base case is quite constrained, e.g., many generators have narrow physical active/reactive power limits, and the angle range of several phase shifters is very small. Furthermore the reactance of more than hundred very short lines has been set to a minimum default value of $0.0002 \mathrm{pu}$ in order to avoid severe ill-conditioned problems.

The system comprises: 9241 buses, 1445 generators, 5546 loads, 14124 lines, 2237 transformers, and 79 PSTs.

A summary of the characteristics of this system according to the voltage level is given in Table II. 


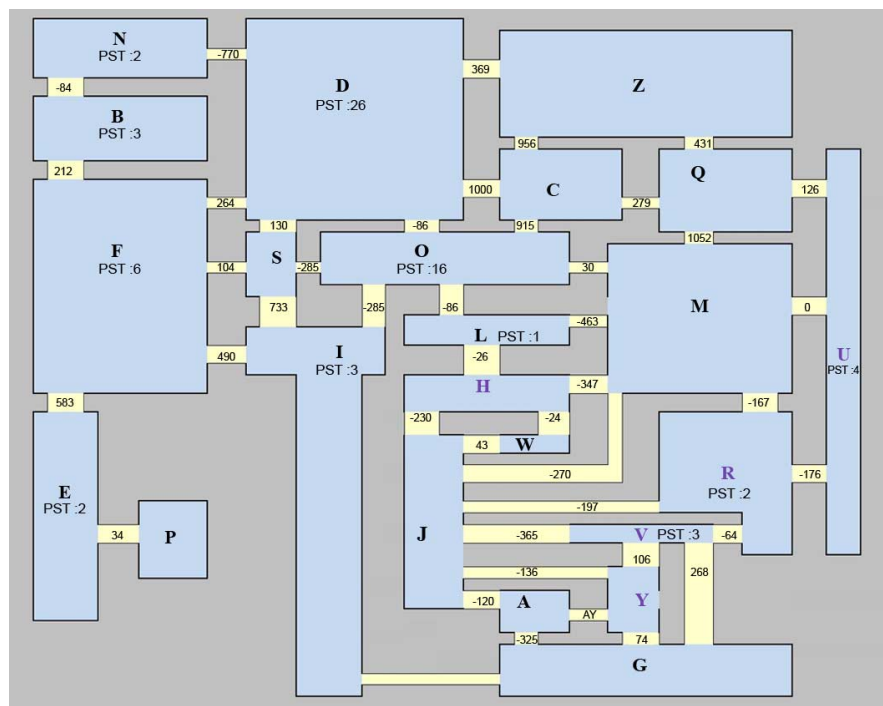

Fig. 2. Top view of the 9241 European system.

Fig. 2 gives a top view of the European system, the power flows exchanged at the base case between countries, and the location of PSTs.

All numerical results and CPU times are given for the following configuration:

- two processors Intel Xeon E5640 4 cores 8 threads CPU 2.66 Ghz 64 bits ram 24 Go cache size $12 \mathrm{MB}$;

- version 7.3 of Hyper Xpress.

\section{B. Overcoming Computational Issues: Effect of Network Reduction and XPRESS Solver Options}

In order to render our approach tractable computationally we rely on an errorless grid reduction and investigate appropriate options for the XPRESS solver.

Let us consider the outage of a line which carries 47 MW. Table III provides the objective function, the computational time, and the overall uncertainty for two grid models: the whole model (i.e., 9241 nodes) and the reduced model of 4067 nodes (this is composed of the set of countries $\{N, B, F, D, S, O, I, C, Z\})$, and for two values of the number of threads in XPRESS. The objective of the benchmark BLV problem is $0.8125 \mathrm{pu}$ and decision variables work properly as no action on the 79 PSTs is taken. Note that, after eliminating 5174 nodes of the external systems, the BLV converges to the same objective function value and no change of discrete variables is observed, which proves an excellent agreement with the simulation that uses the whole model. It is noteworthy to observe that, for the same accuracy of results, the computational time is roughly reduced by a factor of 5 thanks to the network reduction and further by a factor of 2 thanks to the proper choice of the number of threads in XPRESS.

In the sequel of the paper, unless otherwise specified, we model in detail only the internal system, which is composed of the set of countries $\{N, B, F, D, S, O, I, C, Z\}$ and which considers 4067 buses, whereas the external nodes are eliminated by the Ward reduction method.
TABLE III

EFFECT OF NETWORK REDUCTION AND NUMBER OF THREADS IN XPRESS

\begin{tabular}{ccccc}
\hline $\begin{array}{c}\text { number of } \\
\text { buses }\end{array}$ & $\begin{array}{c}\text { number of } \\
\text { threads }\end{array}$ & $\begin{array}{c}\text { BLV objective } \\
\text { function value }\end{array}$ & $\begin{array}{c}\text { computational time } \\
\text { (seconds) }\end{array}$ & $\begin{array}{c}\|s\|_{1} \\
\text { (MW) }\end{array}$ \\
\hline 9241 & 1 & 0.8125243907 & 2345 & 10727 \\
9241 & 4 & 0.8125243897 & 1070 & 10729 \\
4067 & 1 & 0.8125265698 & 386 & 10718 \\
4067 & 4 & 0.8125265735 & 220 & 10722 \\
\hline
\end{tabular}

TABLE IV

Computational Time (s) OF FiXed Point Algorithm FOR COVERING A SINGLE CONTINGENCY

\begin{tabular}{|c|c|c|c|c|}
\hline iteration & \multicolumn{2}{|c|}{ number of threads = 1 } & \multicolumn{2}{c|}{ number of threads = 4 } \\
& SCOPF & BLV & SCOPF & BLV \\
\hline 1 & 9 & 67 & 10 & 54 \\
2 & 10 & 99 & 11 & 48 \\
3 & 10 & 118 & 10 & 51 \\
4 & 10 & 105 & 10 & 49 \\
5 & 10 & 98 & 10 & 59 \\
6 & 10 & 92 & 10 & 51 \\
\hline
\end{tabular}

We further evaluate the effect of the number of threads in XPRESS for the outage of a line which carries $1208 \mathrm{MW}$, which we analyze in detail in the next subsection. When the number of threads is enforced to 1 (respectively to 4 ), the whole process converges in 638 (respectively 373) s. Table IV provides the computational effort of SCOPF and BLV modules at each iteration. One can remark that expectedly the BLV takes significantly more time than the SCOPF, as BLV iterates between several MILP problems whereas the SCOPF solves a single MILP, and that the SCOPF effort is rather insensitive to the number of threads. This significant computational effort fully justifies the proper setting of this parameter. Furthermore, if we adopt the set of countries $\{N, B, D, O\}$ as internal zone, choice which leads to consider only 1734 buses, the whole process converges now in $187 \mathrm{~s} \mathrm{(mipthreads}=4)$.

\section{Contingencies Requiring Preventive Actions}

We consider the outage of a line which carries $1208 \mathrm{MW}$ with respect to the uncertainties induced by the active load power injections in systems $\mathrm{N}$ and $\mathrm{B}$. In our simulations the range of uncertainties is limited only by the set of system constraints in pre-contingency state, which implies that the power balance is satisfied and that the permanent power flow limits $L_{0}$ are met.

Table $\mathrm{V}$ provides the iteration details of the fixed point algorithm and Table IV provides the computational effort of SCOPF and BLV modules at each iteration. One can observe that the worst-case is not controllable only by corrective actions as the objective of the BLV at the first iteration is larger than 1. However, as larger amount of preventive actions are taken at each iteration, the worst-case severity decreases monotonically until the worst-case becomes controllable by preventive actions.

It is worth discussing the underlying mechanism of decision variables in this case. Since the PSTs are assumed in a "flow control" mode, and that immediately after the contingency their monitored flow is below the threshold $L_{1}$ as shown in Fig. 3, the PSTs do not act at all to relieve the overload. However, if we assume that the PSTs are classical remedial actions, they prove being very efficient in removing the overload, as the worst-case 
TABLE V

Fixed PoINT ALgORITHM For COVERING A SingLe CONTINGENCY

\begin{tabular}{ccccc}
\hline $\begin{array}{c}\text { iter } \\
\text { nb }\end{array}$ & $\begin{array}{c}\left\|\hat{u}_{p}-\bar{u}_{p}\right\|_{1} \\
\text { (p.u.) }\end{array}$ & $\begin{array}{c}\text { number of } \\
\text { re-dispatched generators }\end{array}$ & $\begin{array}{c}\text { BLV objective } \\
\text { function value }\end{array}$ & $\begin{array}{c}\left\|u_{p}-\bar{u}_{p}\right\|_{2} \\
\text { (p.u.) }\end{array}$ \\
\hline 1 & 0.0 & 0 & 1.017977 & 0.2501380 \\
2 & 0.056046 & 10 & 1.007202 & 0.2809721 \\
3 & 0.071479 & 8 & 1.002719 & 0.3357288 \\
4 & 0.078853 & 8 & 1.001618 & 0.3550285 \\
5 & 0.082799 & 8 & 1.000082 & 0.3559991 \\
6 & 0.083038 & 8 & 1.000004 & 0.3560181 \\
\hline
\end{tabular}
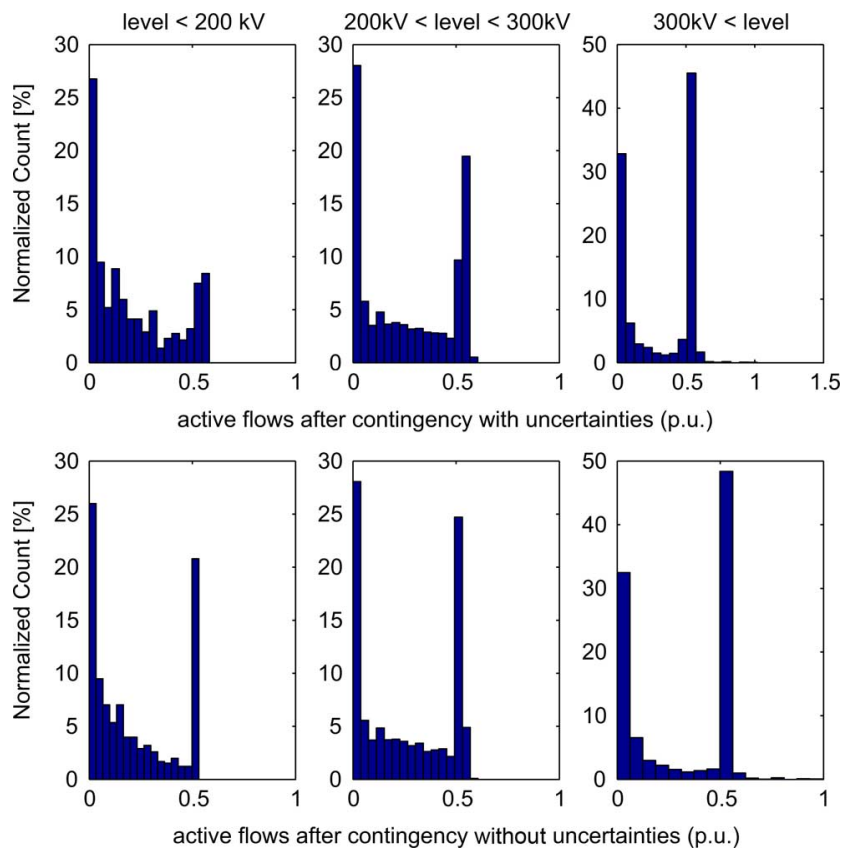

Fig. 3. Active flows distribution.

TABLE VI

CONTINGENCY Clusters

\begin{tabular}{ccccc}
\hline & \multicolumn{4}{c}{ cluster sizes } \\
\cline { 2 - 5 } number of contingencies & $\mathbf{1}$ & $\mathbf{2}$ & $\mathbf{3}$ & $\mathbf{4}$ \\
5126 & 5012 & 5 & 107 & 2 \\
\hline
\end{tabular}

severity computed by the BLV is of only 0.9442 pu at the first iteration thanks to the action of three PSTs.

\section{Contingency Clusters}

In order to obtain contingency clusters, we must decide the number of threads allowed to Xpress for each MILP optimization. From the numerical point of view, experiments on the MIPLIB problems reported in [11] show a significant gain with four threads for cases (like the "protfold" problem) where, during the branch and bound, it becomes increasingly difficult to find better-quality solutions. However, due to the large number of lines or transformers, keeping threads to process the contingencies in parallel seems much more efficient.

Table VI yields the contingency ranking in the four clusters.

In this example, the contingency clusters are determined using the set of countries $\{N, B, F, D, S, O, I, C, Z\}$, while uncertainties are localized on the restricted set $\{N, B, I, C, Z\}$. We take advantage of this configuration by an errorless network reduction.

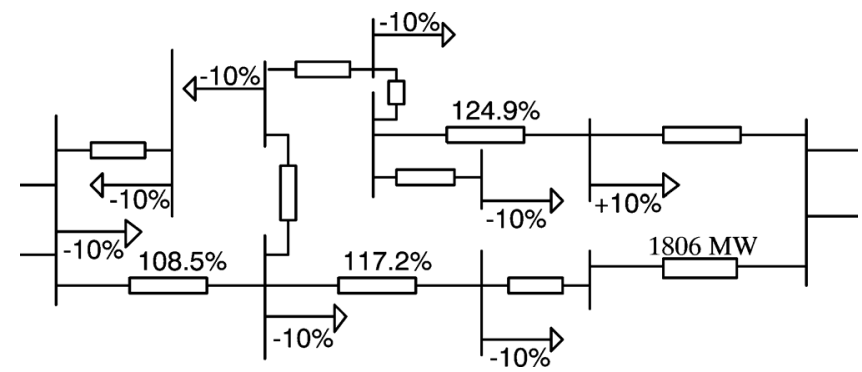

Fig. 4. Sub-network of the PEGASE test case.

TABLE VII

EFFECT OF UNCERTAINTY AREA SIZE ON CONTINGENCY RANKING

\begin{tabular}{cccc}
\hline $\begin{array}{c}\text { uncertainty areas } \\
\text { (countries) }\end{array}$ & $\begin{array}{c}\text { BLV objective } \\
\text { function value }\end{array}$ & $\begin{array}{c}\text { load shedding } \\
\text { (MW) }\end{array}$ & $\begin{array}{c}\|s\|_{1} \\
(\mathrm{MW})\end{array}$ \\
\hline none & 0.938796 & 0 & 0 \\
$N, B, F, D, S, O, I, C, Z$ & 1.000048 & 0 & 10082 \\
all countries & 1.249035 & 1124 & 40597 \\
\hline
\end{tabular}

\section{E. Uncertainty Levels and Contingency Ranking}

We consider a contingency corresponding to the outage of a line which carries $1086 \mathrm{MW}$ in three different scenarios. Note that in each scenario the fixed point algorithm stops after the first iteration due to the following reasons:

- without uncertainties no corrective action is needed (hence the contingency belongs to cluster 1);

- when uncertainties affect a reduced list of countries corrective action on one PST suffices (the contingency belongs to cluster 2);

- when uncertainties affect all countries load shedding is required in SCOPF to ensure system security for the worst uncertainty pattern computed by the BLV (the contingency belongs to cluster 4).

Table VII summarizes the results of these simulations, where the last column measures the level of uncertainty while the BLV objective value measures the uncertainty impact. Uncertainty is of $10 \%$ on active load power. Fig. 4 shows that several lines are significantly overloaded at the solution of the BLV problem corresponding to the last case, which explains why the contingency requires load curtailment to restore security.

\section{CONClusions AND FUtURE WORKS}

To investigate if preventive and corrective controls can maintain the system in a secure state, the method proposed in this paper determines the worst patterns of uncertain variables associated to each contingency. Through discrete bilevel optimization, attention has been focused on the adequate handling of decision variables arising from SO operating rules. Results analysis with the help of clusters reveal whether a contingency is safe or it requires preventive actions. Our results show that these worst cases are characterized by an interaction between uncertainties and the individual flow thresholds which trigger the action of phase shifter transformers. As a consequence, the cluster classification provides also a valuable help for identifying an insufficient coordination of corrective actions.

We prove through numerical simulations the feasibility of the method on very large systems and for a very large number of contingencies. The method is computationally intensive on 
very large systems as it involves the resolution of a significant number of large MILP problems. In this context network reduction, at the expense of a slightly conservative result, is essential to improve the computational effort. The latter depends on the number of contingencies in each cluster, as contingencies that require preventive and corrective actions generally require a more important computational effort than those which require only corrective actions or no action at all. Furthermore, we illustrated the method using a large number of contingencies but usually operators knowledge of the system can be a very efficient pre-filter to reduce the number of potentially dangerous contingencies to some tens/hundreds. The method can be comfortably applied in day-ahead operational planning and even close to real-time for a pre-defined subset of potentially dangerous contingencies.

Further work will concern the improvement of uncertainty models, using historical information about uncertain power injections in a more sophisticated way. As the contingency clusters must be identified for successive anticipated operating states, future work will focus on the reduction of computational effort by properly tuning the parameters of the fixed point algorithms in order to take advantage from the past simulations instead of starting "from the scratch" assumptions. In particular close to real-time the contingency clusters of previous operating points naturally reduce the number of potentially dangerous contingencies to be processed by the algorithm, as the operating point does not generally change dramatically between two clustering analyses.

\section{APPENDIX}

\section{A. Reducing the Problem Size}

In order to reduce the problem size and save CPU time we rely on two observations.

Let us remark that, after the contingency $l$ and before corrective actions, a violation of threshold $L_{1}$ on branch $k$ can only occur if

$$
L_{0, l}\left|D_{k, l}\right| \geq L_{1, k}-L_{0, k}
$$

where the distribution factor $D_{k, l}$ is equal to the flow in the branch $k$ when injections $+\alpha_{l}$ and $-\alpha_{l}$ are applied at the ends $i$ and $j$ of the branch $l$. From the DC approximation equations $B \theta=P$ on the base case, applying the Woodbury formula to the single line outage $l$ of reactance $X_{l}$, the value of $\alpha_{l}$ can be expressed as

$$
\alpha_{l}=\frac{X_{l}}{X_{l}-B_{i, i}^{-1}+B_{i, j}^{-1}+B_{j, i}^{-1}-B_{j, j}^{-1}} .
$$

Due to SO operating rules and condition (15), we can a priori identify the large subset $N(l)$ of corrective actions which will never act for contingency $l$.

Therefore, a correct detection of potential post-contingency violations is ensured by selecting only the branches which verify (17) in the constraints $C_{\text {post }}$ of problem (1):

$$
L_{0, l}\left|D_{k, l}\right|+\sum_{c \in C-N(l)} \max _{\left.u_{c}^{\text {min }}, u_{c}^{\max }\right]}\left|\left(M_{c} u_{c}\right)_{k}\right| \geq L_{1, k}-L_{0, k}
$$

where $C$ denotes the total set of corrective actions. As a consequence, we can identify for each contingency the corrective actions $u_{c}$ and flow limits $L_{1}$ whose modelization is required.

\section{B. Dual Property}

In the DC approximation, when all discrete variables are fixed, the dual variables $\lambda$ of the second-level problem in (9) are solutions of

$$
\begin{aligned}
\max & \left(\lambda_{2}-\lambda_{1}\right)^{T}\left(M_{l} \theta+M_{c} \tilde{e} u_{c}^{m i n}\right)-\tilde{e} \lambda_{4}^{T}\left(u_{c}^{m a x}-u_{c}^{m i n}\right) \\
\text { s.t. } & L_{1}^{T}\left(\lambda_{1}+\lambda_{2}\right) \leq 1 \\
& M_{l}^{T}\left(\lambda_{1}-\lambda_{2}\right) \leq \lambda_{4}-\lambda_{3} \\
& \lambda_{1}, \lambda_{2}, \lambda_{3}, \lambda_{4} \geq 0
\end{aligned}
$$

To replace the bilevel program by a classical linear complementary optimization, the second-level problem of (9) can be expressed by its complementary slackness conditions of the form $x \geq 0, \quad M x+q \geq 0, x^{t}(M x+q)=0$ with $x=$ $\left(\tilde{\delta}_{l}, \tilde{u}_{c}, \lambda_{1}, \lambda_{2}, \lambda_{3}, \lambda_{4}\right)^{T}$, where the matrix $M$ and the vector $q$ depend only on network characteristics.

To solve this classical linear complementary problem through MILP optimization, [12] introduce a new binary variable vector $u$ whose the $i$ th component is equal to one if $x_{i}>0$ and zero otherwise. If the scalar $L$ verifies: $L \geq \max \left(\|x\|_{\infty}, \| M x+\right.$ $\left.q \|_{\infty}\right)$, the following conditions $x \leq L u, M x+q \leq L(1-u)$ are equivalent to $x^{t}(M x+q)=0$ since $x \geq 0$ and $M x+q \geq 0$.

The specific form of (9), (18) implies that each optimal solution $x$ is a priori bounded, providing that every component of the vectors $\tilde{e}, L_{1}, L_{2}, u_{c}^{\max }-u_{c}^{\min }$ is strictly positive and that $\theta$ is feasible for the leader, since the optimal value of the second-level problem is lower than the maximum threshold ratio $L_{2} / L_{1}$. So a sufficiently large constant $L$ can be determined before the MILP optimization.

\section{REFERENCES}

[1] P. Panciatici, Y. Hassaine, S. Fliscounakis, L. Platbrood, M. OrtegaVazquez, J. L. Martinez-Ramos, and L. Wehenkel, "Security management under uncertainty: from day-ahead planning to intraday operation," in Proc. IREP Symp., Buzios, Brazil, 2010.

[2] D. Gan, X. Luo, D. V. Bourcier, and R. J. Thomas, "Min-max transfer capability of transmission interfaces," Int. J. Elect. Power Energy Syst., vol. 25, no. 5, pp. 347-353, 2003.

[3] F. Capitanescu and T. V. Cutsem, "Evaluating bounds on voltage and thermal security margins under power transfer uncertainty," in Proc. PSCC Conf., Seville, Spain, Jun. 2002.

[4] F. Capitanescu, S. Fliscounakis, P. Panciatici, and L. Wehenkel, "Dayahead security assessment under uncertainty relying on the combination of preventive and corrective controls to face worst-case scenarios,' in Proc. PSCC Conf., Stockholm, Sweden, Aug. 2011. [Online]. Available: http://orbi.ulg.ac.be/simple-search?query=capitanescu.

[5] S. Carl and S. Heikkila, Fixed Point Theory in Ordered Sets and Applications: From Differential and Integral Equations to Game Theory. New York, NY, USA: Springer Science, 2011.

[6] A. Mitsos, "Global solution of nonlinear mixed-integer bilevel," $J$. Glob. Optim., vol. 47, no. 4, pp. 557-582, Aug. 2010.

[7] L. Platbrood, S. Fliscounakis, F. Capitanescu, P. Panciatici, C. Merckx, and M. Ortega-Vazquez, Deliverable D3.2: Development of Prototype Software for System Steady-State Optimization of the European Transmission System PEGASE Project, 2011. [Online]. Available: http:// www.fp7-pegase.eu/.

[8] F. Capitanescu, S. Fliscounakis, P. Panciatici, and L. Wehenkel, "Cautious operation planning under uncertainties," IEEE Trans. Power Syst., vol. 27, no. 4, pp. 1859-1869, Nov. 2012. 
[9] B. Ward, "Equivalent circuits for power-flow studies," AIEE Trans., vol. 68 , pp. 373-382, 1949.

[10] A. J. Monticelli, M. V. P. Pereira, and S. Granville, "Security-constrained optimal power flow with post-contingency corrective rescheduling," IEEE Trans. Power Syst., vol. PWRS-2, no. 1, pp. 175-182, Feb. 1987.

[11] R. Laundy, M. Perregaard, G. Tavares, H. Tipi, and A. Vazacopoulos, "Solving hard mixed-integer programming problems with Xpress-MP: A MIPLIB 2003 case study," INFORMS J. Comput., Apr. 2009.

[12] C. Audet, P. Hansen, B. Jaumard, and G. Savard, "Links between linear bilevel and mixed 0-1 programming problems," J. Optim. Theory Applicat., vol. 93, no. 2.

Stéphane Fliscounakis received the M.Sc. degree in applied mathematics from Université Paris Pierre et Marie, Paris, France, and the M.Sc. degree in industrial automation and control from Université Paris Sud Orsay, Paris.

Since 1992, he has worked for RTE, Versailles, France, as a research engineer.

Patrick Panciatici (M'11) received the degree from the French Ecole Supérieure d'Electricité in 1984.

He joined EDF R\&D in 1985, managing the EUROSTAG Project and the CSVC project. He joined RTE in 2003 and participated in the creation of the department "Methods and Support". He is the head of a team which develops real time and operational planning tools for RTE, and ensures operational support on the use of these tools.

Mr. Panciatici is a Member of CIGRE, SEE, and the R\&D ENTSO-E Working Group. He is RTE's representative in PSERC and several European Projects (PEGASE, OPTIMATE, TWENTIES, etc.).

Florin Capitanescu received the degree in electrical power engineering from the University "Politehnica" of Bucharest, Bucharest, Romania, in 1997 and the Ph.D. degree from the University of Liège, Liège, Belgium, in 2003.

His main research interests include optimization methods, in particular optimal power flow, and voltage stability. He works currently as research associate with SnT, University of Luxembourg.

Louis Wehenkel received the degree in electrical engineering (electronics) and the Ph.D. degree from the University of Liége, Liége, Belgium, in 1986 and 1990, respectively.

He is currently a full Professor of electrical engineering and computer science with the University of Liége. His research interests lie in the fields of stochastic methods for systems and modeling, optimization, machine learning and data mining, with applications in complex systems, in particular large-scale power systems planning, operation and control, industrial process control, bioinformatics, and computer vision. 